\title{
"Killing courtesy": Le Songe ou la courtoisie mise en pièce(s)
}

Nathalie Vienne-Guerrin

\section{Citer ce document / Cite this document :}

Vienne-Guerrin Nathalie. "Killing courtesy": Le Songe ou la courtoisie mise en pièce(s). In: XVII-XVIII. Bulletin de la société d'études anglo-américaines des XVIle et XVIIle siècles. №55, 2002. pp. 27-49;

doi : 10.3406/xvii.2002.1795

http://www.persee.fr/doc/xvii_0291-3798_2002_num_55_1_1795

Document généré le 24/05/2016 


\title{
"KILLING COURTESY" : LE SONGE OU LA COURTOISIE MISE EN PIÈCE(S)
}

\begin{abstract}
"Pretty soul, she durst not lie / Near this lack-love, this kill-courtesy" (2.2.88): ${ }^{1}$ voilà en quels termes Puck, maladroit redresseur de tort, s'attendrit sur le sort de Hermia qu'il prend pour Héléna, victime selon lui d'un "sans-amour," d'un "bourreau de la courtoisie."2 Cette scène est une illustration de l'infernale comédie des erreurs mise en œuvre dans le Songe. Puck, aveugle parmi tant d'autres, mauvais déchiffreur de signes, confond ici les couples et prend pour du dédain ce qui est en fait un signe de courtoisie. Le terme doit être ici envisagé dans ses multiples sens. On peut en effet lire dans la distance qui sépare les deux amoureux endormis une simple marque de respect, un geste élégant importé de la cour ou encore un témoignage d'amour courtois. La méprise de Puck apparaît au spectateur de façon d'autant plus criante que, quelques vers plus tôt, Hermia avait rappelé à Lysandre son attachement au respect des codes de bonne conduite:
\end{abstract}

But, gentle friend, for love and courtesy,

Lie further off, in human modesty.

Such separation as may well be said

Becomes a virtuous bachelor and a maid,

So far be distant; and good night sweet friend. (2.2.62-66)

Lysandre avait retrouvé le droit chemin en acceptant de s'étendre à une distance bienséante de sa belle. Le contresens de Puck est porteur d'ironie. Si l'on considère le contexte dans lequel il s'inscrit, il apparaît en effet que le serviteur d'Obéron n'a pas tout à fait tort. L'Athénien dont il est question vient de déroger aux bonnes manières en faisant à Hermia une proposition indécente (2.2.47-58) et l'erreur de Puck ne fait que révéler à contretemps le sort odieux que Lysandre réserve à son aimée.

1. Édition de référence: William Shakespeare, A Midsummer Night's Dream, ed. Peter Holland (Oxford: Oxford UP, 1994).

2. Shakespeare, Le Songe d'une nuit d'été, trans. Jean-Michel Déprats (Arles: Actes Sud: 1990) 35 . 
Au-delà d'un effet ironique immédiat, cet épisode a d'autres résonances. Il suggère en effet que dans la nuit sombre de la forêt les codes de la courtoisie sont remis en cause. Ils se brouillent, se renversent et deviennent illisibles, rendant ainsi l'identité des courtisans elle-même indéchiffrable. Aussi voit-on se dessiner, à la lueur de ce contresens, le monde cauchemardesque du Songe où Shakespeare joue sans cesse sur les décalages entre l'émission et la réception des signes et où le beau geste devient cruauté, la brutalité douceur, le mot d'amour injure.

Notre propos est de montrer comment ce voyage au bout de la nuit ébranle et "défigure" la courtoisie, qui finit par hanter la pièce de son absence-présence. On peut en effet lire le parcours des amoureux comme un retour à l'état de nature qui se mesure à la perte des repères et des codes courtois, c'est-à-dire à la transgression des règles de civilité, à l'abandon des belles manières apprises à la cour, abandon qui va de pair avec une distorsion caricaturale et parodique de l'amour courtois.

Mais si la courtoisie est mise en pièces dans le Songe et son tapage nocturne, c'est pour mieux resurgir, sous des formes grotesques et incongrues, à la "cour des petits" sur laquelle règne Titania mais également dans la pièce mise en scène par les artisans-courtisans. Bottom et ses compères n'ont de cesse, en effet, d'exprimer leur souci de ne pas offenser la noblesse. Ils s'efforcent de montrer qu'ils ont non seulement l'art mais aussi la manière en mettant tout en cuvre pour que leur spectacle ne soit pas discourtois. Ainsi reconstruite, la courtoisie devient un mirage, une illusion, un esprit qui contredit la lettre: le Prologue de Quince, ce mariage monstrueux de l'injure et du compliment, apparaît alors comme l'expression parfaite de cette courtoisie mise en pièce(s).

\section{1. "If you were civil and knew courtesy..." (3.2.147)}

"Est courtois, étymologiquement, ce qui appartient à la cour": 3 si l'on considère un instant le sens étymologique du mot "courtoisie," il apparaît qu'en quittant la cour de Thésée, en passant de la culture à la nature, les amoureux abandonnent objectivement du même coup la courtoisie. Dès le début de la pièce Lysandre et Hermia s'opposent à la cour et à ses bonnes manières en refusant de voir à travers les yeux d'autrui. "I know not by what power I am made bold" (1.1.59), déclare Hermia, mettant ainsi sa révolte sur le compte d'une puissance supérieure, irrationnelle. Dès cet instant, Hermia déroge aux codes de courtoisie tels qu'ils sont, par exemple, détaillés, enseignés dans le traité de John Della Casa intitulé

${ }^{3}$. Étienne Souriau, Vocabulaire d'esthétique (Paris: PU de France, 1990) 520 (article "courtois, courtoisie"). 
Galateo et sous-titré Or Rather, A Treatise of the Manners and Behaviours, it Behoveth a Man to Use and Eschewe, in His Familiar Conversation. A Worke Very Necessary \& Profitable for All Gentlemen, or Other. Publié en anglais en 1576, l'ouvrage contient, entre autres, la recommandation suivante:

I call them fromward people, which will in all things be overtwhart to other men: as the very worde it selfe doth shewe. For, fromewarde, signifieth asmuche, as shorne against the wooll. Now, how fit a thinge this frowardnes is, to wyn the good will of men, and cause men to wyshe well unto them: that your self may easily judge, in that it consisteth in overtwharting other mens desiers: which qualitie never maynteineth friendship, but maketh friends become foes. And therefor let them that desire to be well thought of and welcome amongst men, endevour themselves to shunne this fault: for it breedes no good lyking nor love, but hatred and hurt. I would councell you rather to measure your pleasures by other mens willes: where there shal come no hurt nor shame of it: and therein alwayse to doe \& to saye, more to please other mens myndes and fansies, then your owne. ${ }^{4}$

La confrontation entre Hermia et Thésée semble faire écho à cette évocation d'un manquement aux belles manières. Hermia, la réfractaire ("froward"), défie les règles de la cour. Elle prend le risque de déplaire en refusant de "conformer ses désirs à la volonté"5 des autres ("To fit your fancies to your father's will" [1.1.118]). En matière d'amour, il n'est point de courtoisie qui tienne:

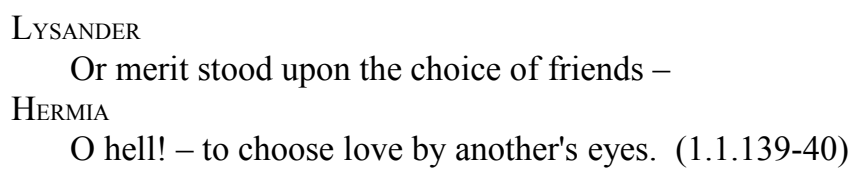

Le duo stichomythique des amoureux contrariés contient ce rejet des manières de la cour qui constituent un carcan auquel l'amour refuse de se soumettre. Leur séjour dans les bois peut être interprété comme un retour à l'état "brut" ("rude" [3.2.262]), un cauchemar où la courtoisie s'égare, laissant apparaître les êtres sous un jour sauvage ("fierce" [3.2.82, 3.2.325, 4.1.68]).

Derrière les bonnes manières évoquées par le mot "courtesy" se cache aussi toute une philosophie de la vie, "une certaine manière de vivre et de penser, élégante et noble," fondée sur des "manières polies, à la fois

${ }^{4}$. John Della Casa, Galateo, 1576 (New York: Da Capo P, 1969) 25. Voir aussi "They should apply them selves to the fancies and minds of their friendes" (29) qui rappelle 1.1.56-57, 1.1.118 et 1.1.140.

5 . Déprats 16. 
gracieuses, franches et affables, avec souvent une certaine recherche du beau geste"; il implique "le désintéressement," "l'importance donnée à l'affectivité et à une certaine culture des sentiments," "la valorisation de la

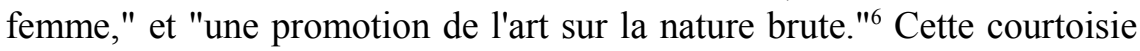
s'apprend dans les livres. Les ouvrages qui lui sont consacrés ("courtesy books"7) à la Renaissance reflètent une culture qui permet à l'individu de se forger lui-même, de se sculpter une identité et une personnalité. Prise dans ce sens humaniste, il semble bien que, dans le bruit et la fureur de cette nuit d'été, la courtoisie soit vouée à se dissoudre ("dissolve," "melt" [1.1.245]), emportant avec elle l'identité des amoureux qui deviennent méconnaissables.

Dans A Marxist Study of Shakespeare's Comedies, Elliot Krieger s'emploie à défendre l'idée stimulante que, si les amoureux fuient les conventions sociales, c'est pour mieux les reconstruire par eux-mêmes hors d'Athènes, substituant ainsi à une loi objective des contraintes qu'ils s'imposent à eux-mêmes: "While trying to get beyond one set of laws, the formal Athenian laws, the lovers - both couples - stay within the confines of social laws, the laws of decorum and propriety." que la courtoisie est souvent évoquée tout au long de la pièce, à la fois par les fées, les artisans, les nobles et les amoureux. ${ }^{10}$ Cependant, notre propos est de montrer que dans la nuit du Songe, cette courtoisie devient fantôme, transformant ainsi les amoureux en fantômes d'eux-mêmes.

\section{La courtoisie fantôme}

Si les couples errants évoquent les bonnes manières, c'est avant tout pour en souligner la fragilité et l'absence. L'image de la courtoisie est mobilisée sur un mode hypothétique qui met en relief son cruel défaut: "I thought you lord of more true gentleness" (2.2.138); "If you were civil and knew courtesy / You would not do me thus much injury" (3.2.14748); "If you were men as men you are in show / You would not use a gentle lady so" (3.2.151-52); "None of noble sort / Would so offend a virgin (3.2.159-60); "If you have any pity, grace or manners / You would

\footnotetext{
${ }^{6}$. Souriau 520-21.

7. Voir John Anthony Cuddon, Dictionary of Literary Terms and Literary Theory (London: Penguin,1991) 200-202.

8. "Am not I Hermia? Are not you Lysander?" (3.2.273)

9. Elliot Krieger, A Marxist Study of Shakespeare's Comedies (London: Macmillan, 1979) 37-61; A Midsummer Night's Dream, New Casebooks, ed. Richard Dutton (London: Macmillan, 1996) 41.

${ }^{10}$. Voir "courteous" (3.1.155, 5.1.176), "Courtesy" (2.2.62, 2.2.83, 3.2.147, 5.1.249), "courtesies" (3.1.165).
} 
not make me such an argument" (3.2.241-42); "Have you no modesty, no maiden shame, / No touch of bashfulness?" (3.2.285-86). Si l'image de la courtoisie et de la noblesse affleure dans la forêt du Songe, c'est pour mieux être niée et défiée. Comme le souligne Cesar Lombardi Barber, à propos de la Nuit des Rois, dans un chapitre intitulé "liberty testing courtesy," ${ }^{11}$ la courtoisie est ici mise à l'épreuve de la liberté.

Les mots de Démétrius qui inaugurent l'arrivée des amoureux dans la forêt semblent bien donner le ton et révéler que l'amour dans cette pièce va se faire discourtois: "I love thee not, therefore pursue me not" (2.1.188). Par cette nuit d'été, le vernis de la courtoisie ne peut que s'écailler. Il n'est plus question ici de cultiver les bonnes manières. Il n'est plus temps de "parler gentiment" ("speak fair" [2.1.199]); l'heure est aux mots enflammés ("passionate words" [3.2.220]), aux "paroles furieuses,"12 à "l'humeur farouche"13 ("fierce vein" [3.2.82]). L'acte 2 met en scène une étape transitionnelle dans un parcours qui mène le quatuor de la courtoisie à l'injure. Shakespeare y met en tension la force brutale et les codes courtois. Le dialogue entre Héléna et Démétrius (2.1.188-244) fait émerger la menace et la tentation d'un retour à l'état brut. "Tempt not too much the hatred of my spirit" (2.1.211): lorsque Démétrius menace Héléna, celle-ci se réfugie derrière les barrières courtoises ("Your virtue is my priviledge" [2.1.220]). Mais quelques vers plus tard, elle souligne que les codes ont été transgressés en déclarant: "Your wrongs do set a scandal on my sex" (2.1.240) ou encore "We should be wooed and were not made to woo" (2.1.242). Au cours de la scène suivante, le débat auquel se livrent Lysandre et Hermia reproduit cette mise en tension de la nature et de la culture. Face à la résistance de Hermia ("Nay, good Lysander / . . . Do not lie so near" [2.2.49-50]), Lysandre a recours au verbe élégant et au mot d'esprit pour habiller son écart de conduite et le rendre présentable. La courtoisie se révèle ici un artifice qui ne trompe pas la vertueuse Hermia. Cette dernière insiste sur le fait qu'elle est une jeune fille bien élevée: "Much beshrew my manners and my pride / If Hermia meant to say Lysander lied" (2.2.60-61). Dans les deux scènes, les femmes se font les gardiennes de la courtoisie tandis que les hommes la menacent. Ce sont les femmes qui semblent le plus longtemps résister à l'épreuve. Dès leur arrivée dans la forêt, Lysandre et Démétrius oublient les bonnes manières, l'un en proposant à Hermia de faire couche commune, l'autre en menaçant Héléna de viol (2.2.214-19). Le parcours

\footnotetext{
11. Voir Cesar Lombardi Barber, Shakespeare's Festive Comedy (Princeton: Princeton UP, 1959) 248-57.

12. C'est ainsi que Jean-Michel Déprats traduit "passionate words" (51).

13. Déprats 46.
} 
des femmes, quant à lui, va de la retenue courtoise à la violence verbale. La scène 2 de l'acte 3 se développe en effet sur le mode de la gradation. Hermia, énonciatrice, domine les divers degrés de la violence verbale. Elle se retient un instant ("Now I do but chide" [3.2.45]) avant de se laisser aller à l'injure ("curse" [3.2.46]). Le barrage de la courtoisie cède lorsqu'elle déclare: "Out, dog; out, cur. Thou driv'st me past the bounds / Of maiden's patience" (3.2.65-66). Hermia "dépasse ici les bornes" de la courtoisie tout comme Héléna le fait un peu plus tard:

HeLENA

What, will you tear

Impatient answers from my gentle tongue?

Fie, fie, you counterfeit, you puppet, you! (3.2.286-88)

Comme le suggère le terme "tear," la douceur ("gentle") courtoise est ici mise en pièces. L'injure est présentée comme une transgression des règles de courtoisie. Dans un paragraphe intitulé "Gentle wordes in communication," John Della Casa fait au lecteur la recommandation suivante: "You must accustome your selfe, to use suche gentle and courtious speache to men, and so sweete, that it may have no maner of bitter taste." ${ }^{14}$ Force est de constater que les mots échangés lors de cette nuit de folie ont un goût amer ("bitter breath" [3.2.44]). Les insultes qui ponctuent toute cette scène sonnent le glas de la courtoisie.

\section{Le tapage nocturne ou le flyting sauvage}

Dans le Songe, la discorde amoureuse fait grand bruit. On entend déjà ce bruit lorsque Titania reproche à Obéron son humeur querelleuse en utilisant le terme "brawl" ("But with thy brawls, thou hast disturbed our sport" [2.1.87]). La dimension sonore des querelles auxquelles se livrent les amoureux apparait également lorsque Obéron déclare: "The noise they make / Will cause Demetrius to awake" (3.2.116). Le terme "noise," dérivé du mot français "noise" qui renvoyait à l'origine à un "bruit, un tumulte," puis à "une querelle, une dispute," associe en lui-même la querelle et le bruit. ${ }^{15}$ Puck, quant à lui, utilise le terme "jangling" pour évoquer ces dissonances nocturnes ("As this their jangling I esteem a sport" [3.2.352-53]). Cet impact sonore est adroitement souligné dans le film de Reinhardt et Dieterle (1935) où les mots d'injure sont multipliés, en écho, par la voix de Puck: "Hang off, thou cat, thou burr; vile thing, let loose, / Or I will shake thee from me like a serpent" (3.2.260-61); "Out,

14. Della Casa 84.

${ }^{15}$. À ce sujet, voir Kenneth Gross, Shakespeare's Noise (Chicago: Chicago UP, 2001) 209. 
tawny Tartar, out; / Out, loathèd med'cine; O hated potion, hence" (3.2.263-64); "Get you gone, you dwarf, / You minimus of hind'ring knotgrass made, / You bead, you acorn" (3.2.328-30). Ces chapelets d'injures déjà généreux sont encore grossis par cet effet d'écho. Dans un article intitulé "Flyting in Shakspere's Comedies," Margaret Galway souligne que, dans le Songe, les échanges d'injures (flyting) dérogent aux règles qui régissent l'affrontement verbal entre l'homme et la femme dans les autres comédies shakespeariennes: "the one exception to Shakespeare's observation of social etiquette in the flytings between lords and ladies occurs in A Midsummer Night's Dream." ${ }^{16}$ Dans Beaucoup de bruit pour rien ou Peines d'amour perdues, la guerre des sexes se construit sur un mode paradoxalement courtois, c'est-à-dire par voie de sous-entendus, de jeux de mots, de traits d'esprit. La violence verbale est en quelque sorte "polie," lissée par l'insinuation. Il n'en est rien ici, où les injures sont des plus directes et ne se cachent pas derrière le jeu d'esprit, provoquant ainsi la réaction d'Hermia: "Why are you grown so rude?" (3.2.262). Dans le Songe, l'injure est sans fard. Hermia se voit "défigurée" ("disfigured") pour devenir un monstre; elle est métamorphosée ("translated") par le biais des insultes. ${ }^{17}$ Le corps est en effet au cœur de ces invectives qui traduisent combien, pour Lysandre, Hermia est devenue une source de dégoût, un objet qui agresse les sens, la vue ("tawny Tartar," "Ethiope"), le toucher ("thou cat," "thou burr," "serpent") et le goût ("hated potion," "loathed med'cine").

Les règles du flyting sont transgressées à bien d'autres égards au cours de cette scène. Forme ritualisée de combat verbal, le flyting se "joue" normalement à un contre un, et il est fondé sur l'alternance des répliques. Ce schéma est ici bouleversé puisque Hermia ne répond pas aux injures de Lysandre mais prend Héléna comme cible de substitution. Si l'on analyse le fonctionnement de cette guerre des sexes, il apparaît que l'injure y est rarement réciproque: Démétrius agresse Lysandre (3.2.259), Lysandre agresse Hermia (3.2.269), Hermia agresse Héléna (3.2.282) qui finit par lui répondre directement une fois et une seule ("You counterfeit, you puppet" [3.2.288]). Héléna préfère en effet la diffamation à l'injure, puisqu'elle attaque Hermia à la troisième personne en prenant les hommes à témoin (3.2.299-305, 323-25). À travers Hermia et Héléna, Shakespeare met en scène deux types de mauvaises langues - celle qui insulte et celle

\footnotetext{
16. Margaret Galway, "Flyting in Shakspere's Comedies," Shakespeare Association Bulletin 10.4 (1935): 185.

${ }^{17}$. À ce sujet, voir Desmet Christy, "Disfiguring Women with Masculine Tropes. A Rhetorical Reading of A Midsummer Night's Dream, A Midsummer Night's Dream: Critical Essays, ed. Dorothea Kehler (London: Routledge, 2001) 299-329, 313-14.
} 
qui diffame -, qui sont bien sûr à jamais bannies des manuels de courtoisie. Les échanges entre les personnages étant ainsi brouillés, le spectateur s'y perd et ne perçoit plus alors qu'un brouhaha, un tapage nocturne tout à la fois divertissant et douloureux, cruel et infantile, violent et féerique. En puisant ses injures dans le lexique de la nature, végétale et animale, Lysandre donne en effet à la discorde des résonances féeriques. En devenant "gland de chêne" ("acorn"), "ronce"18 ("burr"), Hermia se voit intégrée à la cohorte des fées qui entourent Titania ("Peaseblossom," "Cobweb," "Mote," Mustardseed"). L'évocation récurrente de sa petite taille ("minimus of hindering knot-grass made," "bead") la projette dans le monde miniature des fées. L'interpénétration des mondes se manifeste ici par le truchement de l'injure qui engendre tout à la fois la monstruosité et la féerie.

Dans le prolongement de cette violence verbale, on voit se profiler la figure spectaculaire de la mégère. "You juggler, you canker blossom, / You thief of love" (3.2.282-83): Hermia qui s'efforçait d'affirmer ses belles manières à l'acte 2 devient une "jalouse," 19 une "furie" qui se défend bec et ongles (3.2.298):

\section{HeLENA}

$\mathrm{O}$, when she is angry she is keen and shrewd.

She was a vixen when she went to school,

And d though she be but little, she is fierce. (3.2.325)

Héléna est pour beaucoup dans cette métamorphose. Après avoir montré Hermia sous un jour idéal en se remémorant des souvenirs d'enfance, elle la transforme ici en virago.

Dans Henry $I V$, les échanges d'injures entre Falstaff et Hal dessinent une sorte de querelle des gros et des maigres; ici les échanges entre Hermia et Héléna constituent une querelle des grands et des petits. Au "you puppet" (3.2.288) répond le "thou painted maypole" (3.2.296), expression à double tranchant qui inscrit l'injure dans une logique carnavalesque et festive. Cependant, si pour "l'auditeur" cet affrontement verbal est un divertissement ("sport"), il est en revanche une souffrance pour les "acteurs." C'est en jouant sur ce décalage de perception que Shakespeare fait de cette scène une "drôlerie très tragique" ("very tragical

${ }^{18}$. Déprats 52. "Burr" est plus précisément le fruit de la bardane; muni de feuilles à crochets, il s'accroche aux vêtements et désigne en anglais familier la personne collante, le "crampon" dont on n'arrive pas à se défaire. Alexander Schmidt en donne la definition suivante dans son Shakespeare Lexicon in Two Volumes (New York: Dover, 1971) 1: 154: "The rough head of the burdock."

${ }^{19}$. Il semble qu'étymologiquement la mégère soit "celle qui refuse, la jalouse." Voir Dictionnaire historique de la langue française, 2 vols. (Paris: Robert, 1993). 
mirth" [5.1.57]) fondée sur la superposition de l'humeur festive et sérieuse. Ce brouillage du ludique et du sérieux apparaît notamment lorsque Hermia se demande s'il s'agit d'une plaisanterie: "Do you not jest?" (3.2.265).

Dans le flyting traditionnel, les mots ne sont pas censés faire mal. ${ }^{20}$ Mais ici les injures ont ce qu'Evelyne Larguèche appelle "l'effet injure."21 La réaction d'Hermia montre qu'elle est blessée par les attaques de Héléna et Lysandre:

Puppet? Why so? Ay, that way goes the game.

Now I perceive that she hath made compare

Between our statures; she hath urged her height,

And with her personage, her tall personage,

Her height, forsooth, she hath prevailed with him -

And are you grown so high in his esteem

Because I am so dwarfish and so low?

How low am I, thou painted maypole? Speak,

How low am I? I am not yet so low

But that my nails can reach unto thine eyes. (3.2.289-98)

L'injure est d'autant plus mal digérée qu'elle est "spécifique," 22 c'est-àdire qu'elle évoque un trait physique de l'insulté et n'est pas un simple projectile verbal dépourvu de signification.

La scène centrale du Songe suit un parcours qui mène des mots aux actes. Hermia et Héléna sont sur le point d'en venir à l'affrontement physique, ${ }^{23}$ tandis que Démétrius et Lysandre se lancent des défis (3.2.254-55) que l'on oublierait presque tant ils sont "couverts" par le tapage ambiant. En ayant recours au duel, les deux hommes tentent de

${ }^{20}$. Keir Elam décrit l'esprit du flyting en ces termes: "The decisive characteristics of the flyting-sustained vituperation, direct statement on the part of the man, sarcastic insinuation on the part of the woman, etc. - are themselves merely secondary illocutionary rules, but the overriding and implicit convention, that which makes the contest a worthy spectacle, is that the insults should be wittily and inventively varied. It is this witty inventiveness, and not the vilification as such, that is on show" (Keir Elam, Shakespeare's Universe of Discourse: Language-games in the Comedies [Cambridge: Cambridge UP, 1984] 9).

${ }^{21}$. Voir Évelyne Larguèche, l'Effet injure (Paris: PUF, 1983). Dans cet ouvrage qui adopte une approche pragmatique de l'injure, on trouve la distinction entre le mot d'injure (qui peut être inoffensif et ludique) et "l'effet injure." Dans des contextes différents, le même mot peut avoir "l'effet injure" ou pas.

${ }^{22}$. Évelyne Larguèche distingue "l'injure spécifique" de "l'injure non spécifique." L'une fait référence à une caractéristique de l'injurié, l'autre pas.

23. Dans la version de Michael Hoffman (1998), par exemple, les deux femmes finissent par se battre comme des "chiffonnières" dans la boue, ce qui rappelle d'ailleurs les bains ("ducking") réservés aux mégères à l'époque élisabéthaine. 
réinscrire la querelle dans des codes, de la "polir," de l'encadrer. Cependant, en s'insultant, les deux adversaires transgressent l'une des règles essentielles du duel qui, de "fair quarrel," devient "brawl ridiculous." ${ }^{24}$ D'autre part, tout cadre est voué à disparaître dans un monde où Puck l'insaisissable fait la loi. D'abord désamorcé par l'intervention de Hermia qui s'accroche désespérément au cou de Lysandre, le duel est ensuite escamoté par Puck, qui en bon ventriloque vient brouiller la communication entre les deux hommes en leur volant leur voix. ${ }^{25}$ Par l'intervention de Puck, la violence courtoise qu'est censé être le duel se dissout en injures multipliées, décuplées par voie/voix d'écho (3.2.401-30). Les insultes que Puck lance à la place de Démétrius et de Lysandre sont les illustrations les plus flagrantes des injures fantômes qui habitent le Songe.

\section{L'injure fantôme}

Les injures du Songe sont d'autant plus spectaculaires qu'elles côtoient des manifestations enflammées d'amour courtois. La femme est idéalisée ("O Helen, goddess, nymph, perfect, divine!" [3.2.137-38]), on se bat pour elle, on lui fait des serments, on lui jure allégeance ("and then end life when I end loyalty" [2.2.69]), on lui sacrifie sa vie ("And run through fire I will for thy sweet sake" [2.2.109]). La dégradation côtoie l'idéalisation de la beauté, rendant ainsi cette idéalisation caricaturale, monstrueuse, discordante, incongrue. En juxtaposant l'injure et la déclaration d'amour enflammée ("My love, my life, my soul, fair Helena" [3.2.246]) qui partagent la même rhétorique inflationniste, Le Songe met en scène la confusion ou la proximité de la laideur et de la beauté. Dans cette pièce, même le mot d'amour est reçu comme une injure. Héléna ne parvient à voir dans les mots d'amour des deux hommes qu'une injure à son sexe et à elle-même (3.2.218-19). Outrancier, le blason pétrarquisant par lequel Démétrius célèbre, à son réveil, la beauté d'Héléna (3.2.13744) devient insultant ("injury" [3.2.148]). Les serments et les compliments ("To vow and swear and superpraise my parts" [3.2.153]), par leur excès, basculent dans le domaine de l'injure. La courtoisie, qu'il s'agisse de l'amour courtois médiéval ou des bonnes manières, est

${ }^{24}$. Voir la pièce de Middleton et Rowley, A Fair Quarrel. L'expression "Brawl ridiculous" est tirée de Henry $V, 4^{\mathrm{e}}$ Prologue, v. 51. Pour une analyse plus précise de ces codes, voir Vienne-Guerrin, "La réécriture des codes de duel dans l'injure shakespearienne," Réécritures, ed. Jean-Pierre Maquerlot (Rouen: PU de Rouen, 2000) 37-53.

25. À ce sujet, voir Maurice Hunt, "The Voices of A Midsummer Night's Dream," Texas Studies in Literature and Language 34.2 (1992): 218-38. 
devenue illisible: même lorsqu'elle est présente, elle n'est pas perçue comme telle. Au royaume du Songe, l'injure, elle aussi, est fantôme, créée de toutes pièces par l'imagination d'Héléna, pour qui les louanges deviennent injures ("injury," "offend"), moqueries et dérision ("scorn," "disdain," "mockery," "derision"). ${ }^{26}$

Si l'injure nourrissait la passion d'Héléna (2.1.202-10), le compliment a, en revanche, sur elle un "effet injure" qui envahit toute la scène. Ainsi, lorsque Lysandre appelle de ses vœux le "tendre jour" ("Come, thou gentle day" [3.2.418]), il souligne du même coup que la nuit n'a pas été "tendre" mais plutôt cruelle et brutale ("rude"). Certains metteurs en scène signifient cette mise en pièces de la courtoisie en montrant la désagrégation progressive des habits ("apparel") portés par les jeunes Athéniens qui finissent en lambeaux. ${ }^{27}$ La boue qui défigure les amoureux dans la version théâtrale de Robert Lepage $(1992)^{28}$ ainsi que dans les versions filmiques de Peter Hall (1968) et de Michael Hoffman (1998) répond au même dessein. Mais tandis que les amoureux sont mis à nu, les artisans quant à eux, et notamment Bottom, prennent des allures de courtisans.

\section{2. "We are to utter sweet breath" (4.2.38)}

La dernière consigne que Bottom, le meneur d'hommes, donne à ses compagnons avant la représentation de Pyrame et Thisbé est "And, most dear actors, eat no onions nor garlic, for we are to utter sweet breath, and I do not doubt but to hear them say it is a sweet comedy" (4.2.37-39). Cette recommandation, qui renvoie aux conditions réalistes de la représentation, peut être entendue de façon métaphorique. Il s'agit pour les artisans de dire des mots qui seront doux ("sweet breath") aux oreilles

26. Voir "injury" (3.2..148, 219), "injurious" (3.2.195), "offend" (3.2.160), "derision" (3.2.123, 159, 197, 370) "scorn" (2.2.130; 3.2.123, 124, 126, 216, 221, 222, 247), "disdainful" (2.1.261, 2.2.136), "mock" (3.2.150, 156, 299), "mock'ry" (2.2.129), "mockers" (3.2.168).

27. C'est le cas dans la version filmique de Michael Hoffman (1998) où les amoureux finissent à moitié nus, débraillés, décoiffés. Dans la version de Max Reinhardt et William Dieterle on trouve la même dégradation de la tenue des personnages (coiffures et vêtements). On observait la même évolution dans la mise en scène de Stanislas Nordey, Théâtre des Amandiers, Nanterre, 1995.

28. Pour une description de ce Mudsummer Night's Dream, voir Jay L. Halio, "A New Watershed? Robert Lepage's Mudsummer Night's Dream," Shakespeare's Sweet Thunder: Essays on the Early Comedies, ed. Michael J. Collins (Newark: U of Delaware P, 1997) 216-27. 
de la noblesse. Nous ne sommes pas loin ici de la "gentle communication" recommandée par Della Casa. ${ }^{29}$

\section{Bottom et les "manuels" de courtoisie}

Si l'on voit dans la courtoisie un art de plaire cultivé par le courtisan, il est certain que les artisans font tout pour acquérir cet art en devenant des artisans-courtisans. Les hommes aux mains rugueuses ("hard-handed men" [5.1.72]) veulent se faire aussi lisses que possible. Durant la distribution des rôles, Bottom se propose de "rugir gentiment" ("gently" [1.2.74]), afin de ne pas effrayer ces dames. Pour le convaincre de jouer le rôle de Pyrame, Peter Quince lui décrit le personnage en ces termes: "You can play no part but Pyramus; for Pyramus is a sweet-faced man; a proper man as one shall see in a summer's day; a most lovely, gentlemanlike man. Therefore you must needs play Pyramus" (1.2.7679). En jouant Pyrame, Bottom "prend sur lui" (1.2.38) le rôle d'un noble et devient gentleman. Ceux que Puck appelle les "rude mechanicals" (3.2.9) ${ }^{30}$ les "mal polis" veulent faire acte de courtoisie en offrant leur pièce à Thésée et à sa cour. Pour ce faire, d'hommes d'action ils deviennent hommes de lettres. Lorsque Quince propose de mettre leur pièce "en action" ("we will do it in action" [3.1.4-5]), Bottom interrompt le mouvement pour dire "There are things in this comedy of Pyramus and Thisbe that will never please. First, Pyramus must draw a sword to kill himself, which the ladies cannot abide. How answer you that?" (3.1.811). Bottom pratique ici l'auto-censure en se transformant en "Maître des Menus Plaisirs" ("Master of the revels"). ${ }^{31}$ À Starveling qui suggère de supprimer purement et simplement la scène du suicide ("I believe we must leave the killing out" [3.1.14]), Bottom répond que, pour bien faire ("to make all well" [3.1.15]), il faut la mettre en mots:

Write me a prologue, and let the Prologue seem to say we will do no harm with our swords, and that Pyramus is not killed indeed; and for the more better assurance, tell them that I, Pyramus, am not Pyramus, but Bottom the weaver. This will put them out of fear. (3.1.15-20)

Bottom propose ici de rédiger une "assurance-vie," car, comme l'ont souligné de nombreux commentateurs, c'est la crainte d'être pendu ("and

${ }^{29}$. Della Casa 84.

30. Pour une analyse détaillée des résonances de cette expression, voir Patricia Parker, "Rude mechanicals," Subject and Object in Renaissance Culture, ed. Margreta de Grazia, Maureen Quilligan, Peter Stallybrass (Cambridge: Cambridge UP, 1996) 43-82. Voir aussi Parker, Shakespeare from the Margins: Language, Culture, Context (Chicago: U of Chicago P, 1996) 83-115.

31. Voir Richard Wilson, "The Kindly Ones: The Death of the Author in Shakespearean Athens," Dutton, ed. 198-222. 
that were enough to hang us all" [1.2.69]) qui incite la troupe à ménager son public. ${ }^{32}$ Quand la volonté d'être courtois émane de la peur, l'art de plaire que constitue la courtoisie se dévalue en art de ne pas déplaire. Le contenu du Prologue, pour absurde qu'il puisse paraître, résume parfaitement la nature d'un théâtre où l'on ne tue que le nom de Pyrame. Comme le suggère Lysandre, au théâtre, seul le nom passe par l'épée ("O, how fit a word / Is that vile name to perish on my sword" [2.2.112-13]). Pour rendre la violence courtoise, il faut l'habiller de mots qui fonctionnent alors comme des amortisseurs. La deuxième objection soulevée par Snout, puis par Bottom, concernant le spectacle insoutenable que représenterait l'intervention d'un lion sur scène appelle la même solution:

SNOUT

Therefore another prologue must tell he is not a lion. Воттом

Nay, you must name his name, and half his face must be seen through and he himself must speak through, saying thus, or to the same defect: "ladies," or "fair ladies, I would wish you," or "I would request you," or "I would entreat you not to fear, not to tremble. (3.1.31-38)

On voit se profiler ici un deuxième prologue qui prend la forme d'une adresse on ne peut plus courtoise aux dames de l'assistance. Une fois de plus, il est question de faire acte de courtoisie en habillant de mots choisis une réalité trop brutale. Ainsi la pièce Pyrame et Thisbé, telle qu'elle est jouée à la cour de Thésée, déborde de prologues. L'action est enrobée successivement par les deux prologues de Quince, les autoportraits de Snout-le Mur, puis de Snug-le Lion, et de Starveling-le Clair de Lune. C'est par cet enrobage que les artisans entendent purger leur pièce de sa "grossièreté de mortel."

\section{"Purging mortal grossness" (3.1.151)}

Lorsque Titania accueille Bottom dans son monde, elle se donne une mission: "And I will purge thy mortal grossness so / That thou shalt like an airy spirit go" (3.1.151-52). L'expression "mortal grossness" évoque la

32. À ce sujet, voir Theodore B. Leinwand, "'I Believe We Must Leave the Killing Out': Deference and Accommodation in A Midsummer Night's Dream," A Midsummer Night's Dream: Critical Essays, ed. Dorothea Kehler (London: Routledge, 2001) 145-78. Voir aussi Barbara Freedman, "Dis/Figuring Power. Censorship and Representation in $A$ Midsummer Night's Dream," Kehler, ed., 179-215. Voir aussi Nicholas Visser, "'Some satire, keen and critical': Genre and Perspective in A Midsummer Night's Dream," Shakespeare in Southern Africa 9 (1996): 66-74. 
matérialité de la nature humaine, comparée à l'évanescence et la légèreté du monde des fées, mais elle sugère aussi que le séjour de Bottom à la cour de Titania fait partie de l'entreprise de "polissage" que constitue le parcours des artisans dans Le Songe. La cour de Titania peut être vue comme une version déformée de la cour de Thésée. Certains metteurs en scène soulignent d'ailleurs cet effet de miroir en distribuant aux mêmes acteurs les rôles de Thésée et Obéron et les rôles de Hippolyta et Titania. Dans les mains de Titania, Bottom devient un gentilhomme ("gentle mortal" [3.1.130]) qui a tous les attributs de la grâce: la voix ("note" [3.1.131]), le corps ("shape" [3.1.132]), la vertu ("fair vertue" [3.1.133]), l'esprit ("I can gleek upon occasion" [3.1.139]). Titania enjoint à sa cohorte de serviteurs de se montrer courtois envers son protégé:
Be kind and courteous to this gentleman.
Hop in his walks, and gamble in his eyes.
Feed him with apricots and dewberries,
With purple grapes, green figs, and mulberries;
The honeybags steal from the humble-bees,
And for night tapers crop their waxen thighs
And light them at the fiery glow-worms' eyes
To have my love to bed, and to arise;
And pluck the wings from painted butterflies
To fan the moonbeams from his sleeping eyes.
Nod to him, elves, and do him courtesies. (3.1.155-65)

Pour la Reine des Fées, être aimable et courtois ("kind and courteous") signifie servir, nourrir, monter la garde du sommeil de Bottom et lui faire des révérences. Envisagée sous un autre angle, cette liste de recommandations suggère que la courtoisie implique une maîtrise de la nature, que l'on façonne et défigure à sa guise. Être courtois, c'est ici contrarier la nature. La scène qui suit nous fait apercevoir Bottom tout à son art de la conversation. C'est à sa conversation que l'on reconnaît le gentleman, le parfait courtisan. Bottom se révèle un maître en matière de civilité. Ses répliques sont ponctuées de "your worships" (3.1.170-71), "good master" (3.1.173-74), "gentleman" (3.1.175), "I pray you" (3.1.177), "I beseech you" (3.1.180). Ces démonstrations de courtoisie se poursuivent à l'acte 4 , au cours d'une scène où Titania polit littéralement Bottom par ses caresses et ses mots doux:

TitANiA

Come, sit thee down upon this flowery bed,

While I thy amiable cheeks do coy,

And stick musk-roses in thy sleek smooth head,

And kiss thy fair large ears, my gentle joy. (4.1.1-4) 
"Amiable," "sleek," smooth," "gentle," sont autant de termes qui suggèrent le "po-lissage" que subit Bottom. L'artisan montre ses belles manières en multipliant les "monsieur," "good monsieur" et autres "Cavalierie," se conformant ainsi à certaines des consignes dictées dans The Book of the Courtier de Castiglione. ${ }^{33}$ Cependant, tout en cultivant l'art de la courtoisie, Bottom en dénonce également l'incongruité. "Pray you, leave your courtesy, good monsieur" (4.1.20): en enjoignant à Monsieur Mustardseed de ne pas faire de "courbettes," Bottom signale que les artifices de la courtoisie sont ici déplacés. Il souligne le décalage entre le texte et son contexte, entre les mots et ceux qui les prononcent. Quand la courtoisie se fait "courtis-ânerie," elle ne peut que s'effondrer au moment même où elle se fabrique, tout comme le mur si civil ("courteous") puis si vil ("vile") qui sépare Pyrame de Thisbé.

\section{"Thanks, courteous wall" (5.1.176)}

Le fait que toutes les belles manières que nous venons de décrire soient le fait de personnages appelés Bottom, Peaseblossom, Mustardseed, Cobweb ou Mote suffit à les rendre "malséantes." Il ne sied pas plus à un âne d'être gentilhomme qu'à un mur d'être courtois. Et pourtant, dans le Songe, les murs, les ânes, les lions et même la lune sont courtois. Ils le sont et ne le sont pas. "Here come two noble beasts in: a man and a lion" (5.1.215-16); "a very gentle beast" (5.1.225); "Truly, the moon shines with a good grace" (5.1.261-62): les commentaires ironiques des membres de l'assistance soulignent et effacent d'un même souffle la courtoisie "mise en pièce" (de théâtre) par les artisans. Le parcours du Mur dans Pyrame et Thisbé est emblématique des mécanismes de renversement qui habitent le Songe:
And thou, O wall, O sweet, O lovely wall,
That stand'st between her father's ground and mine,
Thou wall, O wall, O sweet and lovely wall,
Show me thy chink, to blink through with mine eyne.
Wall shows his chink
Thanks, courteous wall. Jove shield thee well for this.
But what see I? No Thisbe do I see.
O wicked wall, through whom I see no bliss,
Cursed be thy stones for thus deceiving me. (5.1.171-79)

\footnotetext{
33. "I would have our Courtier to speake and write in that sorte, and not onely choose gorgeous and fine wordes out of every part of Italie, but also I would judge him worthie praise to use some of those terms both French and Spanish, which by our custome have beene admitted" (Baldassare Castiglione, The Book of the Courtier, trans. Thomas Hoby [London: Dent, 1944] 57).
} 
En un instant, de charmant le mur devient "méchant." La louange se change en injure. Cette tirade propose une version à peine grossie des métamorphoses subies par Hermia et Héléna, soumises à la perception des hommes. Au-delà de cet effet de miroir entre le monde de Pyrame et Thisbé et celui du quatuor désaccordé, on peut lire dans cette tirade une réflexion sur la courtoisie. En effet, cette scène suggère que courtoisie rime avec supercherie: "Cursed be thy stones for thus deceiving me" (5.1.179). De courtois le mur devient fourbe. À la lumière de ce texte, le spectateur est amené à explorer la courtoisie dans ses rapports à l'illusion, à la perception et à la réception des signes.

\section{3. "Courtesy killed... with kindness" 34}

Le Prologue de Quince serait sans conteste un très bon Prologue au Songe tant il en exprime l'ambiguïté. En quelques vers, on entrevoit tous les dysfonctionnements, tous les décalages entre l'émission et la réception des signes qui habitent cette pièce et notamment les jeux cruels des amoureux. Il est un commentaire sur les renversements, les distorsions, les errances que nous venons d'examiner. Il est aussi l'incarnation de la "discorde musicale" évoquée à plusieurs reprises par Thésée et Hippolyta ("Musical confusion," "musical discord" [4.1.102-127]; "How shall we find the concord of this discord?" [5.1.60]), au cours de passages qui résonnent comme des commentaires sur le fonctionnement global du Songe. Ce prologue apparemment absurde contient en germe la substantifique moelle de la pièce. Il est la manifestation la plus parfaite de ce que l'on peut appeler les injures courtoises du Songe.

\section{"If we offend, it is with our good will" (5.1.108)}

Les parcours inversés des artisans et des courtisans se croisent à l'acte 5 au cours de la représentation de Pyrame et Thisbé. Nous avons déjà montré comment la courtoisie, prise dans son sens médiéval ou moderne, devenait injure dans le monde bouleversé des amoureux. Le Prologue de Quince est une version condensée de ce renversement.

If we offend, it is with our good will.

That you should think, we come not to offend

But with good will. To show our simple skill,

That is the true beginning of our end.

Consider then we come but in despite.

We do not come as minding to content you,

\footnotetext{
${ }^{34}$. Nous jouons ici sur le titre de la pièce de Thomas Heywood, A Woman Killed with Kindness.
} 
Our true intent is. All for your delight

We are not here. That you should here repent you

The actors are at hand. (5.1.108-116)

Ce texte met en scène le décalage et la contradiction entre l'esprit et la lettre. Le prologue de Quince a ceci de particulier qu'il se dédit ("unsay" [1.1.181]) au moment même où il se dit. Les défauts de ponctuation, qui correspondent à une mauvaise maîtrise du souffle de l'acteur amateur, contrarient l'intention courtoise qui sous-tend ce texte. On peut imaginer que c'est la peur de déplaire, la hantise d'être discourtois et de risquer sa tête, qui expliquent ces défauts de prononciation. Au cours de cette scène "la courtoisie tue la courtoisie" tout comme "la vérité tue la vérité" ("truth kills truth" [3.2.129]). Il appartient alors au public de redresser le corps monstrueux de ce texte ambigu. Nombre de commentateurs soulignent que le compliment se renverse ici pour devenir injure. Mais il convient d'être plus précis. Ce texte n'est pas reçu comme une injure, il n'a pas "l'effet injure." Il est reçu comme un hommage courtois à la cour de Thésée. Ce texte a bien "l'effet courtois" qu'il visait. D'ailleurs Thésée le recueille bien comme un témoignage de courtoisie, comme un signe de bienvenue ("out of this silence yet I picked a welcome" [5.1.100]). Ici se révèle la magie d'un texte métamorphosé par l'oreille qui l'entend. On assiste à la traduction ("translation") d'une injure en compliment. C'est l'auditeur/traducteur, et non l'auteur/énonciateur, qui construit le sens et récrit le texte de Quince. L'oreille qui reçoit recrée la courtoisie ici mise en pièce(s). Le plus étonnant est que ce texte fantôme ainsi imaginé par l'auditeur efface le texte source.

Cependant, quelques critiques ont noté que ce travestissement du texte des artisans pourrait bien révéler une erreur d'interprétation, un contresens de la part de Thésée et de sa cour. ${ }^{35}$ La correction ("amend" [5.1.211]), la rectification du texte pourrait être une erreur. Tout bien considéré, le public fait dire à un texte ce qu'il ne dit pas. L'esprit réoriente, reconstruit la lettre. Mais si l'on s'en tient aux mots, tels qu'ils sont prononcés par Quince, la courtoisie manifestée par la troupe d'acteurs s'en trouve ébranlée et est reléguée au statut de trompe-l'œil, d'illusion. Ce qui est pris pour un hommage peut résonner comme une provocation. Il est possible d'interpréter les erreurs de ponctuation comme une série de lapsus révélateurs. Le texte oscille entre déférence et insulte. ${ }^{36}$ Comme le note Theodore B. Leinwand, ${ }^{37}$ on peut déceler cette

\footnotetext{
35. Voir notamment Leinwand et Visser.

36. "Like Elizabethan artisans who rioted not to offend, but with good will, the prologue teeters back and forth between deference and offensiveness" (Leinwand 154).

37. Leinwand 153.
} 
même ambiguïté lorsque Bottom imagine un Prologue qui semblerait dire ("seem to say") qu'ils ne feront aucun mal avec leurs épées (3.1.15-17). Shakespeare suggère également cette ambivalence en utilisant à deux reprises l'expression "against your/our nuptial" (1.1.125; 5.1.75). Bien sûr, "against" signifie ici "in preparation for," mais si l'on décide de l'entendre d'une oreille plus moderne, l'hommage nuptial se transforme en provocation. Ainsi ce prologue subit une double métamorphose: d'abord déformé par Quince, il est ensuite reconstruit par l'auditeur qui décide d'y entendre un hommage courtois. En éliminant tout un pan du texte, en rayant son contenu injurieux, l'interprétation se fait ici censure.

\section{"Keen mock'ry" (2.2.129)}

Au cours de la représentation de Pyrame et Thisbé, les "acteurs" sont aussi des "auditeurs." Il ne fait aucun doute en effet qu'ils entendent les commentaires ironiques du public. Dans son livre consacré aux bonnes manières, Della Casa bannit la pratique de ce qu'il appelle "scorne" (la raillerie) en ces termes:

Doe not allow, that a man should scorne or scoffe at any man, what so ever he be: no not his very enimy, what displeasure so ever he beare him: for, it is a greater signe of contempt and disdaine, to scorne a man, then to do him an open wrong. . . . And the Nature and effect of a scorne, is properly to take a contentation and pleasure to do another man shame and villany: thoughe it do our selves no good in the world. ${ }^{38}$

Puis il s'emploie à établir la distinction entre ce qu'il appelle "a scorne" et "a mock": "There is no difference betweene a scorne and a mocke: but the purpose alone and intent a man hath, in the meaning the

38. Della Casa 62-63. Il poursuit en ces termes: "So that, good maner \& honesty, would us beware we scorne no man in any case: wherin they be much to be blamed, that reprove men those blemishes they have in their person, eyther in woords, as Master Forese da Rabatta did, laughing at the countenaunce of Master Giotta: or in deeds, as many doe, counterfeting those that stutter, haulte, or be crookte shoulderd. And likewyse, they that scoffe at any man, that is deformed, ill shapen, leane, litle or a dwarfe, as much to be blamed for it: or, that make a gybing and iesting at such follyes as another man speaketh, or the woordes that escape him by chaunce: and with all, have a sporte and a pleasure to make a man blush: all these spitefull behaviours and fashions, worthely deserve to be hated, and make them that use them, unworthy to beare the name of honest gentleman.

And such as use to iest at a man, be very lyke unto these: I mean them that have a good sport to mocke and beguile men, not in spite or scorne, but on a merriment alone" (Della Casa 63-64). 
one the other. For a man mockes and laughes otherwhile, in a sport and a pastime: but his scorn is ever in a rage and disdain. ${ }^{39}$

Selon Della Casa, ce qui différencie la raillerie de la moquerie, c'est l'intention de l'énonciateur. Si la moquerie peut être plaisante et divertissante, la raillerie cause du tort. Si la moquerie est d'essence ludique, la raillerie est sérieuse. Après avoir établi cette distinction, Della Casa note combien il est néanmoins difficile de connaître l'intention de l'autre. "It many times chanceth, in boording and Iesting, one tackes in sporte, the other strykes againe in earnest: and thus from playing, they come to fraying." 40 En matière de moquerie, il convient donc d'être prudent, car une plaisanterie peut aisément tourner à l'injure. La confusion de la moquerie plaisante et de l'injure anime la scène de la représentation et explique le malaise qu'elle peut provoquer chez le spectateur. Les courtisans, par leurs commentaires moqueurs produisent un bruit de cour aussi déplaisant que plaisant. Cette scène peut être lue comme une scène d'injure courtoise. Les remarques acerbes et ironiques de la cour constituent un sous-titrage injurieux et dérangeant. La réaction de Clair de Lune met en relief "l'effet injure" potentiellement présent dans cette scène. Atteint par les réflexions de l'assistance qui parasitent son texte, Clair de Lune, en "pétard" ("in snuff" [5.1.245]), abandonne le vers et finit par expédier sa réplique en prose. $\mathrm{Si}$, dans la forêt, les courtisans pratiquaient l'insulte directe, à la cour, l'injure prend la forme de la moquerie chargée d'ironie: "It is the wittiest partition that ever I heard discourse" (5.1.165-6); "A very gentle beast, and of a good conscience" (5.1.225); "Well roared, Lion" (5.1.259); "Well run, Thisbe" (5.1.260); "Well moused, Lion" (5.1.263). Derrière chacun de ces compliments, le spectateur et l'acteur peuvent percevoir une injure. La moquerie est ici une forme d'injure masquée par le compliment.

À la lumière de cette scène de cour, on comprend mieux la réaction d'Héléna qui ne parvenait à entendre dans les louanges de Démétrius et de Lysandre que de la moquerie et de la dérision: "Wherefore was I to this keen mock'ry born? / When at your hands did I deserve this scorn?" (2.2.129-30); "Good troth, you do me wrong - good sooth, you do - in such disdainful manner me to woo" (2.2.135-36); "O that a lady of one man refused / Should of another therefore be abused!" (2.2.139-40); "Never did mockers waste more idle breath." (3.2.168). Dans The Book of the Courtier, l'une des recommandations faites aux dames est de savoir distinguer le faux amour du vrai: "It were first needfull to teach her to

\footnotetext{
${ }^{39}$. Della Casa 64.

40. Della Casa 65.
} 
know them that make semblant to love, and them that love in deede." ${ }^{41}$ Or on reconnaît l'amoureux au fait qu'il ne parle pas beaucoup: "true lovers as they have a burning heart so have they a colde tongue, with broken tongue and sodaine silence. Therefore (may hap) it were no false principle to say, He that loveth much, speaketh little." ${ }^{42}$ Dame de la cour, Héléna sait que la courtoisie peut n'être que faux-semblant et trompel'œil.

\section{"Let your epilogue alone" (5.1.347)}

Le refus de Thésée d'entendre l'épilogue vient couronner cette scène d'injure courtoise:

No epilogue, I pray you; for your play needs no excuse. Never excuse; for when the players are all dead there need none to de blamed. Marry, if he that writ it had played Pyramus and hanged himself in Thisbe's garter, it would have been a fine tragedy; and so it is, truly, and very notably discharged. But come, your bergamask, let your epilogue alone. (5.1.347-53)

Thésée parle ici une langue aussi double ("double tongue" [2.2.9]) que celle de Quince lorsqu'il prononce son premier Prologue. Après avoir subi le spectacle des artisans par courtoisie ("but yet in courtesy, in all reason, we must stay the time" [5.1.249-50]), il refuse d'entendre l'épilogue proposé par Bottom et en écrit un lui-même, créant ainsi un texte fantôme qui oscille également entre injure et compliment. Cet épilogue imaginaire peut résonner comme une parodie du Prologue de Quince. En effet, il déborde de contradictions et n'est que confusion. En déclarant que la pièce n'a pas "besoin d'excuses," Thésée donne paradoxalement à cet épilogue fictif le contenu d'une excuse, ce qui semble suggérer que les artisans, malgré tous leurs efforts, ont bel et bien offensé leur public et devraient donc s'en excuser. D'autre part, le discours ambivalent de Thésée aboutit à une aporie, lorsque le souverain déclare que cela aurait pu être une bonne tragédie ("it would have been a fine tragedy" [5.1.351]), mais que cela l'a été ("and so it is, truly" [5.1.351-52]). De ces contradictions émergent deux images dérangeantes. "When the players are all dead, there need none to be blamed" (5.1.349): en déclarant que "les comédiens sont tous morts," Thésée confond les acteurs et les personnages que les artisans avaient pourtant pris tant de peine à dissocier. Ce qui pourrait ressembler à un simple lapsus révèle que, pour les comédiens, l'heure a sonné. Aussitôt nés, aussitôt morts:

\footnotetext{
41. Castiglione 237.

42. Castiglione 238.
} 
c'est Thésée qui, par cette phrase, les assassine. La version de Reinhardt et Dieterle suggère cette brutalité: lorsque les comédiens reviennent sur scène pour offrir leur bergamasque, les auditeurs ont quitté la salle pour rejoindre leurs appartements. Le spectacle est amputé, les comédiens enterrés. Après avoir verbalement assassiné les comédiens, Thésée laisse entendre que la mort de l'auteur eût été la cerise sur le gâteau: ${ }^{43}$ "if he that writ it had played Pyramus and hanged himself in Thisbe's garter, it would have been a fine tragedy." On a beau être à la cour, il y a peu de courtoisie, peu de "vertu" dans ce "if."44

En entendant le Prologue, Thésée choisit de prêter attention à son esprit plutôt qu'à sa lettre en préférant le compliment à l'injure. Il est possible que les artisans fassent le même choix à la fin de la pièce. Il est également possible qu'ils fassent le choix contraire. Dans la version de Peter Hall (1968), Peter Quince, l'auteur, apparaît déconfit à l'évocation de sa propre mort. La mise en scène d'Alexandru Darie (1991), quant à elle, mettait au jour toute la dimension injurieuse de cette scène. Dans cette version, Thésée "remerciait" les artisans en leur jetant une bourse, mettant ainsi leur courtoisie "en pièces." Les comédiens, meurtris par les commentaires injurieux de l'assistance, ne ramassaient pas cet argent dont ils avaient pourtant rêvé ("sixpence a day" [4.2.19-22]), mais quittaient la scène sans un bruit, en lançant à Thésée des regards dédaigneux. ${ }^{45} \mathrm{La}$ version de Michael Hoffman (1998) montre combien la réplique de Thésée met le sens en suspens. Les acteurs sont dans la plus complète perplexité, jusqu'à ce qu'ils entendent le "very notably discharged" qui résonne comme un verdict libérateur. Ils laissent alors éclater leur joie et leur soulagement. L'imagination de Bottom et sa troupe censure les mots de Thésée ("imagination amend them" [5.1.211]), "édite" son discours et choisit d'y entendre un hommage. Le discours de Thésée est pourtant sans autre "merci" que celle qui est dictée par l'indulgence princière, pour une pièce qui reste, aux yeux du souverain, une œuvre "grossière" ("palpablegross play" [5.1.358]).

Ainsi, l'oreille qui reçoit donne au discours sa valeur courtoise ou injurieuse. Les mots sont "à prendre" ("idle breath"), dans un sens doux ("sweet breath") ou amer ("bitter breath"). Avant le spectacle, Bottom, confiant, dit à ses compères "I do not doubt but to hear them say it is a sweet comedy" (4.2.39). Cette formulation n'est pas anodine car elle ménage la possibilité d'un décalage entre ce qui est dit ("ils vont dire") et ce que l'on perçoit de ce qui est dit ("nous allons les entendre dire"). Dans

\footnotetext{
43. Pour une approche historique de cette "mort (suicide) de l'auteur," voir Wilson.

${ }^{44}$. Voir Comme il vous plaira, "much virtue in 'if'," 5.4.98.

45 . Voir Shakespeare, ed. Holland 251.
} 
cette béance, qui sépare ce qui est dit de ce qui est entendu, réside l'interprétation. L'épilogue prononcé par Puck fait écho au Prologue de Quince ("if we offend") et à l'épilogue imaginé par Thésée. Au cœur de ces trois textes, on trouve l'évocation d'une offense que l'auditeur devrait pardonner à l'acteur:

If we shadows have offended, Think but this and all is mended:

That you have but slumbered here,

While these visions did appear. (5.1.414-17)

Robin laisse le soin à l'auditeur de corriger lui-même sa perception de l'œuvre. C'est l'auditeur qui se fait le redresseur du tort hypothétique qui lui a été fait ("think but this"). C'est à l'auditeur d'effacer l'injure ("if you pardon, we will mend"). ${ }^{46}$ Les mots de Puck suggèrent également que, lorsque l'on rêve, on a tous les droits, même celui d'être discourtois. Les dérèglements de la courtoisie que nous venons d'évoquer sont étroitement liés à la dimension onirique du Songe. En rêve, les ânes peuvent être des gentilshommes et les courtisans des brutes. Dans Beaucoup de bruit pour rien, Béatrice et Benedick échangent les mots suivants:

BeATRICE

Courtesy itself must convert to disdain if you come in her presence. BENEDICK

Then is courtesy a turncoat. (Much Ado About Nothing 1.1.118-20)

On a l'impression que le Songe est l'incarnation de ces réflexions aphoristiques. Dans la forêt où errent les quatre noctambules, la courtoisie se mue en dédain ("converts to disdain"). La grossièreté, elle, se change en civilité, tout comme la mer évoquée par Obéron s'adoucit en entendant le chant de la sirène ("That the rude sea grew civil at her song" [2.1.52]). De ces "échanges de courtoisie" naît l'harmonieuse discorde d'un Songe dont les "senteurs suaves"47 sont également odieuses: "odious savours sweet" (3.1.77). Le terme "brawl" est emblématique de cette ambiguïté, puisqu'il évoque des disputes mais également des danses plus raffinées et courtoises que ne le sont les rondes orchestrées par Titania. ${ }^{48}$ Le mot "noise"49 est lui aussi pétri de cette ambivalence: en français, "noise" évoquait certes la rudesse des querelles mais il "désignait autrefois toute sorte de bruit, y compris le bruit agréable fait par les

46. À propos de la dimension satirique du Songe, voir Visser.

47. Déprats 40.

48. À ce sujet, voir Skiles Howard, The Politics of Courtly Dancing in Early Modern England (Amherst: U of Massachusetts P, 1998) 70-81.

49. Voir Gross 209. 
violons à une noce, et l'on disait "faire noise" en bonne part au sens de se réjouir." ${ }^{50}$ Le bruit qui précède les noces des Athéniens est fait de ce mélange de courtoisie et de violence.

Nathalie VIENNE-GUERRIN

Université de Rouen

${ }^{50}$. Dictionnaire historique de la langue française 2: 1327. 